\title{
Diffuse pruritic erythema as a clinical manifestation in anti-SAE antibody-associated dermatomyositis: a case report and literature review
}

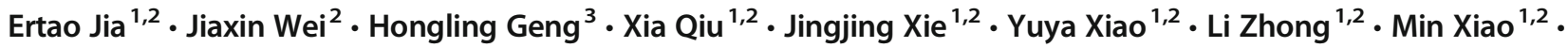 \\ Yanying Zhang ${ }^{1,2} \cdot$ Yubao Jiang $^{1,2}$. Jianyong Zhang ${ }^{1,2}$
}

Received: 10 March 2019 / Revised: 8 April 2019 / Accepted: 12 April 2019 / Published online: 27 April 2019

(C) The Author(s) 2019

\begin{abstract}
Anti-small ubiquitin-like modifier-1 activating enzyme (anti-SAE) antibodies have been recently discovered especially for myosin and identified as dermatomyositis (DM) marker. The frequency of anti-SAE antibodies in DM patients is extremely low. Diffuse pruritic erythema may be one kind of clinical manifestations of DM with anti-SAE antibodies. In this report, a 48year-old female patient with amyopathic dermatomyositis (ADM) carrying anti-SAE antibodies presented diffuse pruritic erythema for 5 months. Diffuse pruritic erythema improved after treatment with prednisolone, cyclosporine, and thalidomide. The clinical characteristics of 75 previously reported cases with anti-SAE antibody-positive DM were reviewed, and the manifestations of the Asian and Western cohorts were compared. It was revealed that the Asian patients were more susceptible to diffuse erythema (17/34 vs. $3 / 41, P=0.000)$, dysphagia ( $16 / 34$ vs. $10 / 41, P=0.040)$, and interstitial lung disease (ILD) $(21 / 34$ vs. $5 / 41$, $P=0.000)$ compared with the Western patients. The frequency of malignancy in the Asian cohort was significantly higher than that in the Western cohort (10/34 vs. $4 / 41, P=0.030)$.
\end{abstract}

Keywords Anti-SAE antibody · Anti-small ubiquitin-like modifier-1 activating enzyme antibody $\cdot$ Dermatomyositis · Diffuse erythema $\cdot$ Literature review $\cdot$ Malignancy

\section{Introduction}

Anti-small ubiquitin-like modifier-1 activating enzyme (antiSAE) antibodies have been recently discovered as myosin-

Ertao Jia, Jiaxin Wei and Hongling Geng contributed equally to this work.

Ertao Jia

sailing1980@126.com

Jianyong Zhang

zhangjianyong2008@126.com

1 The Department of Rheumatology, Shenzhen Traditional Chinese Medicine Hospital, No.1, Fuhua Road, Futian District,

Shenzhen 518033, Guangdong, China

2 The Fourth Clinical Medical College of Guangzhou University of Chinese Medicine, No.1, Fuhua Road, Futian District,

Shenzhen 518033, Guangdong, China

3 The Department of Gynecology, Guangdong Provincial Hospital of Chinese Medicine, The Second Affiliated Hospital of Guangzhou University of Chinese Medicine, Guangzhou 510120, Guangdong, China specific autoantibodies [1] and identified as dermatomyositis (DM) marker. The frequency of anti-SAE antibodies in DM patients is extremely low, ranging from 1.5 to $8.0 \%$. The antibodies are often associated with the initial presentation of amyopathic dermatomyositis (ADM). However, whether diffuse erythema is a kind of clinical manifestations of DM with anti-SAE antibodies remains unclear [2]. In this study, a Chinese patient with anti-SAE antibody-positive ADM who suffered from diffuse pruritic erythema was reported. The clinical characteristics of 75 previously reported cases were reviewed, and the manifestations of the Asian and Western cohorts were compared.

\section{Case presentation}

A 48-year-old female presented diffuse pruritic erythema for 5 months. The erythema was distributed on her scalp, face, neck, chest, and back (Fig. 1a-c). Other skin manifestations included heliotrope rash, Gottron's signs, V-neck sign, shawl 

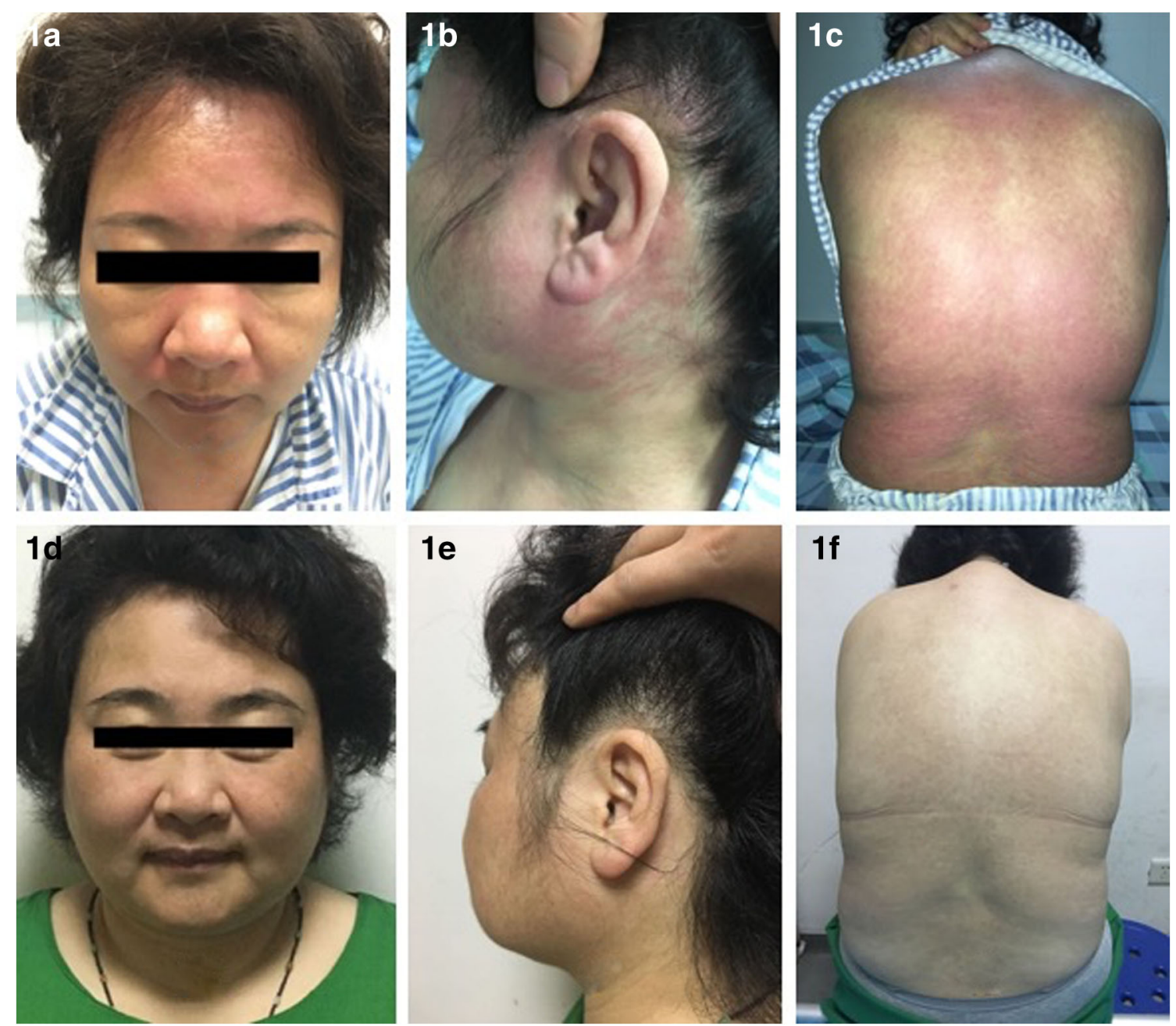

Fig. 1 The diffuse erythema distributed in face (a), scalp (b), and back (c) before treatment. The diffuse erythema was improved in face (d), scalp (e), and back (f) after treatment

sign, periungual lesions, and Mechanic's hands. Muscular weakness, dysphagia, or interstitial lung disease (ILD) was not found. A swollen lymph node was observed on the left occipital. According to the Cutaneous Dermatomyositis Disease Area and Severity Index (CDASI), the total activity score was 33, and total damage score was 4. ANA (1:320), anti-SAE, and anti-SSA/Ro52 were positive, while other myositis-specific antibodies were normal. Creatine kinase (CK), lactate dehydrogenase, aspartate aminotransferase, aspartate aminotransferase, and electromyography were normal. Erythrocyte sedimentation rate (ESR), C-reactive protein (CRP), tumor markers, anti-HBV, anti-hepatitis $\mathrm{C}$ virus (anti-HCV), anti-human immunodeficiency virus (anti-HIV), syphilis antibody, thyroid function, and serum potassium were normal. There was a palpable $2 \mathrm{~cm} \times 2 \mathrm{~cm}$ swollen lymph node on the left occipital bone. Histopathological examination of the skin revealed mild hyperkeratosis, localized atrophy and thinning of the epidermis, denatured collagen fibers of the superficial dermis, and deposition of a small quantity of pigment-like particles, as well as perivascular edema with

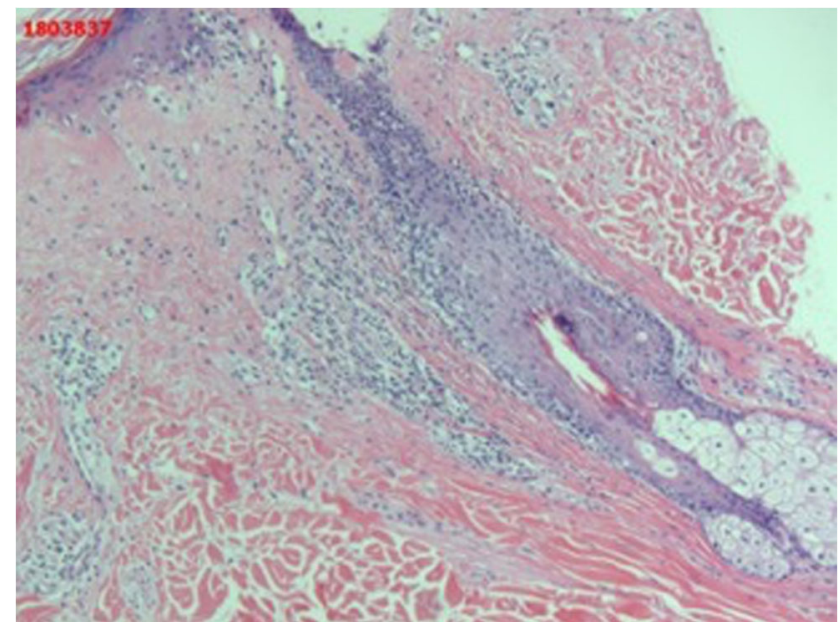

Fig. 2 Pathology of skin tissue biopsy: the epidermis of the skin tissue was slightly hyperkeratotic, the local epidermis atrophied and thinned, the collagen fibers in the superficial dermis were degenerated, and a few pigment-like particles were deposited 


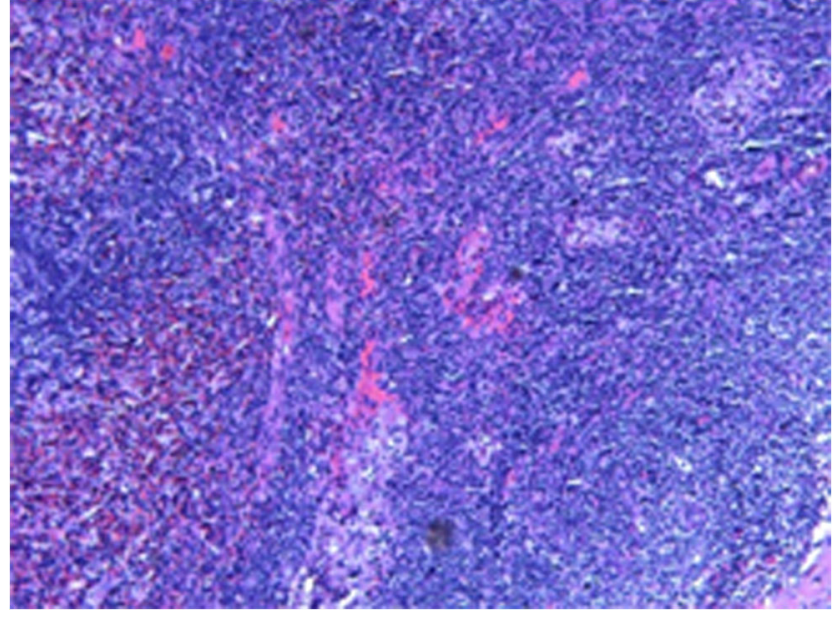

Fig. 3 Pathology of lymph nodes: it was reactive hyperplasia with lymphocytic infiltration

lymphocytic infiltration (Fig. 2). Reactive hyperplasia was confirmed by lymph node pathology (Fig. 3). The patient had been misdiagnosed as eczema and given anti-histamine treatment, while it was not efficient. The pruritic erythema improved after taken $30 \mathrm{mg}$ of prednisolone daily and $10 \mathrm{mg}$ of methotrexate weekly. However, after 6 months, the patient developed hair loss, and then the daily treatment plan changed to $100 \mathrm{mg}$ of cyclosporine and $10 \mathrm{mg}$ of prednisolone. However, the pruritic erythema relapsed. The pruritic erythema improved after adding $50 \mathrm{mg}$ of thalidomide daily for 6 months (Fig. 1d-f). The total activity score was 4 , and total damage score was 0 .

\section{Discussion}

The anti-SAE antibodies are classified into two subtypes, SAE1 and SAE2, with molecular weights of 40 and $90 \mathrm{kDa}$, respectively. A large-scale study on Chinese DM patients found that the frequency of anti-SAE antibody-positive DM was 3\% [3]. The frequency of anti-SAE antibodies in Japanese DM patients was 1.8\% [4], and 8\% in British Caucasian adult DM patients [5]. A research conducted in Italy reported that 5 of 75 patients with DM had anti-SAE antibodies [6]. A Greek research reported the similar frequency (3/44) of anti-SAE in DM [7]. The autoantibody was strongly associated with the HLA-DQB $1 * 03$, HLA-DRB $1 * 04$, and HLA-DQA $1 * 03$ haplotypes [5].

Here, 75 cases of anti-SAE antibody-positive DM have been reported, including the presented case (Table 1). The races have been differentiated based on the literatures. Asian

Table 1 Clinical characteristics and treatment of SAE antibody-positive patients

\begin{tabular}{|c|c|c|c|c|c|}
\hline & Number (\%) & Asian cohort $(n=34)$ & Western cohort $(n=41)$ & $X^{2}$ & $P$ \\
\hline \multicolumn{6}{|l|}{ Clinical characteristics } \\
\hline Skin involvement & $75(100)$ & NA & NA & NA & NA \\
\hline Heliotrope rash & $38(50.7)$ & 23 & 15 & 7.174 & $0.007^{*}$ \\
\hline Gottron's signs & $46(61.3)$ & 28 & 18 & 11.587 & $0.001 *$ \\
\hline V-neck sign & $28(37.3)$ & 17 & 11 & 4.265 & $0.039 *$ \\
\hline Shawl sign & $23(30.7)$ & 18 & 5 & 14.513 & $0.000 *$ \\
\hline Mechanic's hands & $11(14.7)$ & 11 & 0 & 15.545 & $0.000 *$ \\
\hline Periungual lesions & $23(30.7)$ & NA & NA & NA & NA \\
\hline Diffuse erythema & $20(26.6)$ & 17 & 3 & 17.316 & $0.000 *$ \\
\hline Alopecia & $1(1.3)$ & NA & NA & NA & NA \\
\hline Calcinosis & $2(2.7)$ & NA & NA & NA & NA \\
\hline Myopathy & $58(77.3)$ & 22 & 36 & 5.658 & 0.170 \\
\hline Dysphagia & $26(34.7)$ & 16 & 10 & 4.417 & $0.040 *$ \\
\hline Elevation of $\mathrm{CK}$ & $35(46.7)$ & 18 & 17 & 0.984 & 0.321 \\
\hline Lung involvement & $26(34.7)$ & 21 & 5 & 20.164 & $0.000 *$ \\
\hline Arthritis & $4(5.3)$ & NA & NA & NA & NA \\
\hline Malignancy & $14(18.7)$ & 10 & 4 & 4.730 & $0.030 *$ \\
\hline \multicolumn{6}{|l|}{ Treatment } \\
\hline GC (21-39 mg) & 2 & NA & NA & NA & NA \\
\hline $\mathrm{GC}(\geq 40 \mathrm{mg})$ & 4 & NA & NA & NA & NA \\
\hline $\mathrm{GC}+\mathrm{MTX}$ & 1 & NA & NA & NA & NA \\
\hline $\mathrm{GC}+$ tacrolimus & 2 & NA & NA & NA & NA \\
\hline GC + immunoglobulins & 1 & NA & NA & NA & NA \\
\hline $\mathrm{GC}+\mathrm{CYS}$ & 2 & NA & NA & NA & NA \\
\hline $\mathrm{GC}+\mathrm{AZA}$ & 1 & NA & NA & NA & NA \\
\hline $\mathrm{GC}+\mathrm{CYC}+\mathrm{MTX}+\mathrm{CYS}$ & 1 & NA & NA & NA & NA \\
\hline $\mathrm{GC}+\mathrm{CYS}+\mathrm{MTX}+\mathrm{AZA}$ & 1 & NA & NA & NA & NA \\
\hline $\mathrm{GC}+\mathrm{CYS}+$ thalidomide & 1 & 1 & NA & NA & NA \\
\hline
\end{tabular}

Five patients were not illustrated gender. $N A$ not available, $C K$ creatine kinase, $G C$ glucocorticoid, $M T X$ methotrexate, $C Y C$ cyclophosphamide, $C Y S$ cyclosporine, $A Z A$ azathioprine

$* P<0.05$ between Asian cohort and Western cohort 
cohort was major Chinese and Japanese patients, and Western cohort was major Caucasian patients. Except for one case of a male with DM for 5 years, all were adult onset. The female/ male ratio was 1.8:1; however, 5 patients did not describe their gender [6], and there was no significant difference in female/ male ratio between in the Asian (22/12) and the Western (23/ 18) cohorts.

The frequency of Heliotrope rash, Gottron's signs, V-neck sign, Shawl sign and Mechanic's hands was higher in the Asian cohort than the Western cohort. Especially, the frequency of diffuse erythema was substantially higher in the Asian $(50.0 \%)$ cohort than the Western $(7.3 \%)$ cohort, which may reflect differences in ethnicity. Only the patient in our case report suffered from diffuse pruritic erythema. Thus, diffuse erythema should be taken as one kind of cutaneous manifestations of DM into account with anti-SAE antibodies. Skin manifestations preceded muscle involvement (77.3\%) in all cases. There was no difference in myopathy and CK level between the Asian and Western cohorts $(P=0.321)$. The frequency of dysphagia in the Asian cohort was higher than that in the Western cohort. ILD was mainly systemic manifestation (34.7\%). The frequency of ILD in the Asian cohort (61.8\%) was also higher than that in the Western cohort (12.2\%). The frequency of arthritis in all cases was low (5.3\%). In addition, a significantly higher frequency of anti-melanoma differentiation-associated gene 5 (MDA5) antibodies was observed in Chinese patients with polymyositis (PM)/DM than in Japanese patients [8]. These findings suggest that distinct genetic and/or local environmental factors affect Chinese and Japanese patients with PM/DM, who have been considered a homogeneous population in previous studies.

A previous study found that the incidence of cancer in SAE-positive patients was significantly higher than that in anti-SAE-negative patients, $57 \%$ and $12 \%$ [9]. We here found that the incidence of malignancy was $18.7 \%$ (14/ 75). The frequency of malignancy in the Asian cohort [3, 9-11] was remarkably higher than that in the Western cohort (10/34 vs. $4 / 41)[5,6,12]$. The difference in malignancy may be associated with ethnicity as well. Tumors in the Asian cohort were various, including esophageal cancer $(1 / 10)$, colon cancer $(3 / 10)$, rectal cancer $(2 / 10)$, lung cancer $(1 / 10)$, renal cell carcinoma $(1 / 10)$, uterine cancer $(1 / 10)$, and other tumors $(1 / 10)$, in which the incidence of digestive system malignancy was the highest $(6 / 10)$. Ovarian cancer $(1 / 4)$, colon cancer $(1 / 4)$, and other tumors $(2 / 4)$ were reported in the Western cohort. In addition, at least $50 \%$ of patients had tumors within 3 years after the diagnosis of DM. These results indicate that antiSAE antibody in DM patients is a risk factor for malignancy, especially in the Asian cohort.
Glucocorticoid (GC) had been preferred for the treatment of DM. High dose of GC is always combined with immunosuppressive agents (methotrexate, cyclophosphamide, cyclosporine, or cyclosporine) for clinical application. In the presented case, the pruritic erythema improved after adding thalidomide. Thus, we considered that thalidomide may be used to improve diffuse erythema. The majority of anti-SAE antibody-positive patients (78\%) improved after receiving GC and/or immunosuppressive agents.

In this study, diffuse erythema was one kind of cutaneous manifestations of DM with anti-SAE antibodies. The Asian patients may be more susceptible to diffuse erythema, dysphagia, ILD, and malignancy compared with the Western patients.

Acknowledgements The Sanming Project of Medicine in Shenzhen (SZSM201612080), Scientific research project of Traditional Chinese Medicine Bureau of Guangdong Province (20171084).

\section{Compliance with ethical standards}

Disclosure None.

Patient consent and confidentiality The patient signed a written informed consent form for the purpose of publication of results of this case study.

Open Access This article is distributed under the terms of the Creative Commons Attribution 4.0 International License (http:// creativecommons.org/licenses/by/4.0/), which permits unrestricted use, distribution, and reproduction in any medium, provided you give appropriate credit to the original author(s) and the source, provide a link to the Creative Commons license, and indicate if changes were made.

\section{References}

1. McHugh NJ, Tansley SL (2018) Autoantibodies in myositis. Nat Rev Rheumatol 14:290-302

2. Lundberg IE, de Visser M, Werth VP (2018) Classification of myositis. Nat Rev Rheumatol 14:269-278

3. Ge Y, Lu X, Shu X, Peng Q, Wang G (2017) Clinical characteristics of anti-SAE antibodies in Chinese patients with dermatomyositis in comparison with different patient cohorts. Sci Rep 7:188

4. Muro Y, Sugiura K, Akiyama M (2013) Low prevalence of antismall ubiquitin-like modifier activating enzyme antibodies in dermatomyositis patients. Autoimmunity 46:279-284

5. Betteridge ZE, Gunawardena H, Chinoy H, North J, Ollier WER, Cooper RG, McHugh NJ (2008) Clinical and human leucocyte antigen class II haplotype associations of autoantibodies to small ubiquitin-like modifier enzyme, a dermatomyositis-specific autoantigen target, in UK Caucasian adult-onset myositis. Ann Rheum Dis 68:1621-1625

6. Tarricone E, Ghirardello A, Rampudda M, Bassi N, Punzi L, Doria A (2012) Anti-SAE antibodies in autoimmune myositis: 
identification by unlabelled protein immunoprecipitation in an Italian patient cohort. J Immunol Methods 384:128-134

7. Zampeli E, Venetsanopoulou A, Argyropoulou OD, Mavragani CP, Tektonidou MG, Vlachoyiannopoulos PG, Tzioufas AG, Skopouli FN, Moutsopoulos HM (2019) Myositis autoantibody profiles and their clinical associations in Greek patients with inflammatory myopathies. Clin Rheumatol 38:125-132

8. Chen Z, Hu W, Wang Y, Guo Z, Sun L, Kuwana M (2015) Distinct profiles of myositis-specific autoantibodies in Chinese and Japanese patients with polymyositis/dermatomyositis. Clin Rheumatol 34:1627-1631

9. Muro Y, Sugiura K, Nara M, Sakamoto I, Suzuki N, Akiyama M (2015) High incidence of cancer in anti-small ubiquitin-like modifier activating enzyme antibody-positive dermatomyositis. Rheumatology (Oxford) 54:1745-1747

10. Inoue S, Okiyama N, Shobo M, Motegi S, Hirano H, Nakagawa Y, Saito A, Nakamura Y, Ishitsuka Y, Fujisawa Y, Watanabe R,
Fujimoto M (2018) Diffuse erythema with 'angel wings' sign in Japanese patients with anti-small ubiquitin-like modifier activating enzyme antibody-associated dermatomyositis. Br J Dermatol 179: 1414-1415. https://doi.org/10.1111/bjd.17026

11. Haruka Matsuo KY, Umezawa Y, Nakagawa H, Muro Y (2018) Anti-SAE antibody-positive dermatomyositis in a Japanese patient: a case report and review of the literature. J Clin Rheumatol 00:1-2. https://doi.org/10.1097/RHU.0000000000000683

12. Bodoki L, Nagy-Vincze M, Griger Z, Betteridge Z, Szöllősi L, Dankó K (2014) Four dermatomyositis-specific autoantibodiesanti-TIF1 $\gamma$, anti-NXP2, anti-SAE and anti-MDA5-in adult and juvenile patients with idiopathic inflammatory myopathies in a Hungarian cohort. Autoimmun Rev 13:1211-1219

Publisher's note Springer Nature remains neutral with regard to jurisdictional claims in published maps and institutional affiliations. 